SPACE OBSERVATIONS 


\title{
FAR ULTRAVIOLET OBSERVATIONS OF DWARF NOVAE MADE WITH THE HOPKINS ULTRAVIOLET TELESCOPE
}

\author{
KNOX S. LONG \\ Space Telescope Science Institute \\ 3700 San Martin Drive \\ Baltimore, MD 21218, USA
}

\begin{abstract}
Observations with the Hopkins Ultraviolet Telescope on the Astro- 1 and Astro-2 space shuttle missions have provided the first set of moderate ( $3 \AA$ ) resolution far ultraviolet (FUV) spectra of dwarf novae to include the wavelength range between $\operatorname{Ly} \alpha$ and the Lyman limit. Important lines which are detected in the HUT spectra of dwarf novae in this wavelength range include $S$ VI $\lambda \lambda 933,945$, C III $\lambda 977, O$ VI $\lambda \lambda 1032,1038$, $\mathrm{P} \vee \lambda \lambda 1118,1128$ and $\mathrm{C}$ III $\lambda 1176$, as well as the higher order Lyman lines. The observations confirm earlier IUE observations that two dwarf novae U Gem and VW Hyi - have quiescent FUV spectra dominated by the white dwarf, but suggest that the quiescent FUV emission from three other dwarf novae - SS Cyg, WX Hyi and YZ Cnc - are dominated by emission from a hot portion of the disk or a disk corona. The spectra obtained of the dwarf novae Z Cam and EM Cyg in outburst and also of the nova-like variable IX Vel can be modeled reasonably successfully in terms of steady state disks constructed by adding appropriately-weighted stellar model spectra.
\end{abstract}

\section{Introduction}

As a result of the observations made with International Ultraviolet Explorer, tremendous progress has been made in the last fifteen years in terms of characterizing the FUV spectra of disk-dominated cataclysmic variables at wavelengths down to and including Ly $\alpha$. As a result, we now know that (i) the disk, a wind emerging from the inner portions of the system, the hot spot, and the white dwarf (WD) all contribute to the ultraviolet spectra of dwarf novae, (ii) the UV spectrum of a dwarf nova in outburst resembles that expected from an optically thick disk and (iii) the UV outburst is often delayed with respect to the optical outburst implying that, at least in 
some cases outbursts develop in the outer disk [see e.g. reviews by Córdova (1995); Córdova \& Howarth (1987); Drew (1990)]. More recently, HST has been used to to obtain the first spatially resolved maps of accretion disks of CVs in the FUV and to obtain high signal-to-noise ratio, high resolution spectra of the strongest wind lines in several dwarf novae and nova-like systems. [See Horne et al. (1994); Robinson et al. (1995); Mason et al. (1995); Baptista et al. (1995), as well as numerous contributions to this conference.] However, like IUE, HST is limited to wavelengths longward of $\sim 1160 \AA$.

The Hopkins Ultraviolet Telescope consists of a $0.9 \mathrm{~m} \mathrm{f} / 2$ primary feeding a prime-focus Rowland spectrograph with a photon-counting microchannel plate detector. Designed for use on the space shuttle, it was flown on Astro-1 in 1990 December and again in 1995 March. Optimized for the wavelength range between Ly $\alpha$ and the Lyman limit, it was used to obtain $830 \ldots 1860 \AA$ spectra with a resolution of $\sim 3 \AA$ of a wide range of astrophysical objects, including $5 \mathrm{CVs}$ on Astro- 1 and $10 \mathrm{CVs}$ on Astro-2. The only other spectra of CVs which include the wavelength range between Ly $\alpha$ and the Lyman limit were obtained with Voyager, which has a resolution of $18 \AA$ and far less sensitivity (Polidan, Mauche \& Wade 1990), and ORFEUS (Raymond et al. 1995), which has higher resolution, but has been used to observe only three CVs - AM Her, V3885 Sgr and Z Cam. A complete description of HUT and its performance on Astro-1 has been provided by Davidsen et al. (1992); improvements made to the instrument and its performance on Astro-2 are discussed by Kruk et al. (1995).

Here, I will summarize our analysis of the spectra of CVs obtained with HUT on Astro-1 and give an overview of the initial results of an analysis of the spectra obtained of dwarf novae on Astro-2.

\section{Dwarf novae in quiescence}

Five dwarf novae - SS Cyg, YZ Cnc, WX Hyi, U Gem, VW Hyi - were observed in quiescence on Astro-2. Three of these dwarf novae were actually observed several times during the mission, at intervals separated by several days. The time-averaged spectra of each of the dwarf novae are shown in Fig. 1. The spectra of the different dwarf novae differ considerably. U Gem and VW Hyi have spectra which can be characterized in terms of broad Lyman line absorption features and relatively narrow metal absorption lines, while SS Cyg, YZ Cnc, and WX Hyi have relatively flat continua and broad emission lines.

In the 'standard' theory of disks in dwarf novae, quiescent accretion disks are expected to have a temperature of $\lesssim 6000 \mathrm{~K}$ (Meyer \& MeyerHofmeister 1982; Cannizzo, Ghosh \& Wheeler 1982) and are expected to 


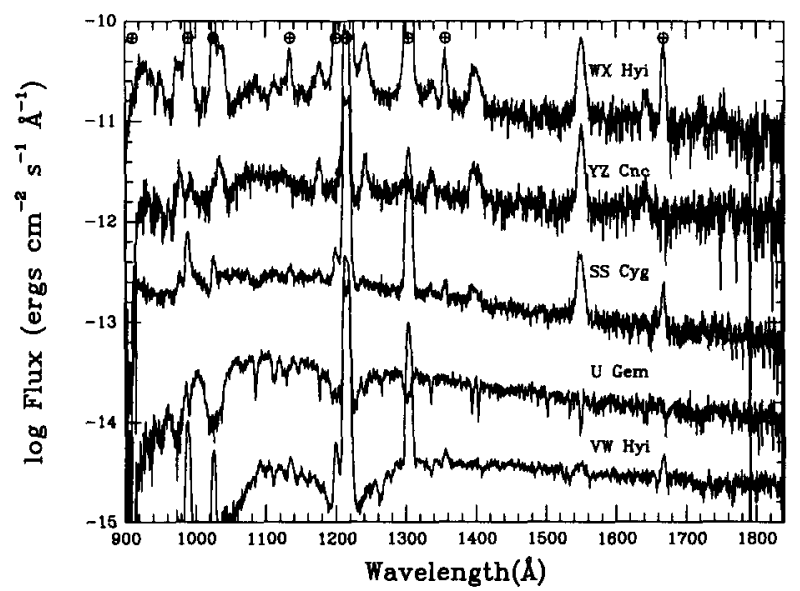

Figure 1. The spectra obtained of dwarf novae in quiescence with HU'T on Astro-2. The spectra have been offset from one another for clarity. Lines which may be airglow are indicated by $\oplus$ on all figures. In the spectra of WX Hyi, YZ Cnc and SS Cyg, in which $\mathrm{O}$ VI $\lambda \lambda 1032,1038, \mathrm{~N} \vee \lambda \lambda 1239,1243$, and $\mathrm{C}$ IV $\lambda \lambda 1548,1551$ are seen in emission, the Lyman lines are apparently absent and the continuum is bluer than in the spectra of $U$ Gem and VW Hyi, in which these same lines are weak or absent.

be optically thin in the UV continuum. As a result, the WD is generally assumed to dominate the FUV light in dwarf novae. WDs have broad Lyman absorption lines as do HUT spectra of U Gem and VW Hyi. The UV flux from U Gem and VW Hyi is also about that expected from a WD at the estimated distances of U Gem and VW Hyi, given estimates of the temperature based on the widths of Ly $\alpha$, as indeed IUE observations had indicated (Panek \& Holm 1984, Mateo \& Szkody 1984). Because the strong gravitational field causes metals to settle into the atmosphere on a time-scale of $1 \ldots 3 \mathrm{yr}$ (cf. Michaud 1987), absorption lines with equivalent widths of $\sim 1 \AA$ are not usually seen in the spectra of isolated WDs, unless the temperatures are very low $(<12000 \mathrm{~K})$ or very high $(>90000 \mathrm{~K})$. However, the time-scale to replenish the $\left(\sim 10^{-12} \mathrm{M}_{\odot}\right)$ atmosphere of a WD is short in a dwarf nova system and therefore prominent metal absorption lines are expected and indeed are observed in both U Gem and VW Hyi. Thus the HUT spectra provide strong support for the conclusions first reached with IUE that the WD dominates the FUV spectra of U Gem and VW Hyi.

U Gem was observed on both Astro- 1 and Astro-2. The Astro-1 observation occurred just $10 \mathrm{~d}$ after the system had returned to optical quiescence following a normal outburst (Long et al. 1993). Model fits to the Astro-1 observations implied an average WD temperature of $\sim 38000 \mathrm{~K}$ and near solar abundances in the WD atmosphere. This was higher than the $30000 \mathrm{~K}$ estimated from IUE spectra far from outburst (Panek \& Holm 1984) and 


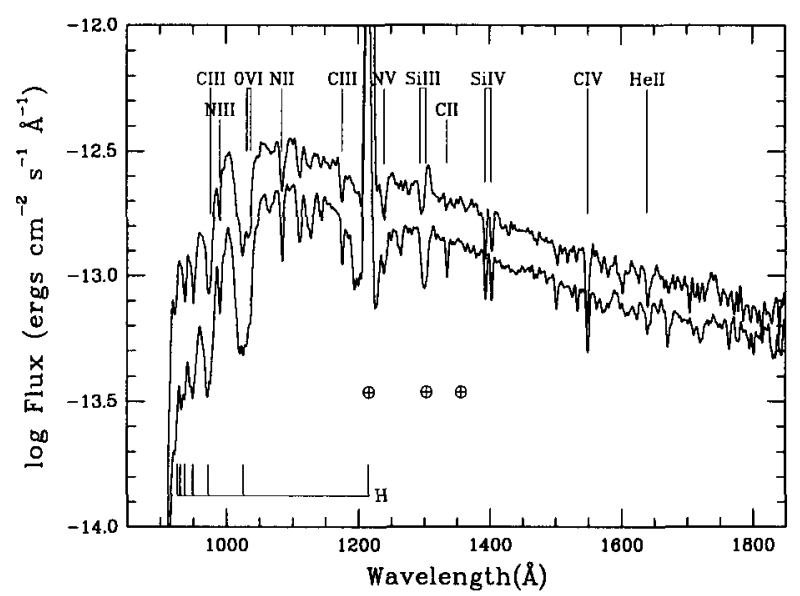

Figure 2. The spectra of U Gem obtained with HUT on Astro-1 (upper curve) and Astro-2 (lower curve). The flux at $1450 \AA$ from U Gem as observed on Astro-2 $185 \mathrm{~d}$ after the return to quiescence was about $30 \%$ less than on Astro-1.

suggested that the WD in $U$ Gem is heated during outbursts and then cools between outbursts, consistent with evidence for a decline in the UV flux with time from outburst (Kiplinger, Sion \& Szkody 1991). The Astro-1 data were also fit to two-temperature models in which $85 \%$ of the surface had a temperature of $30000 \mathrm{~K}$ and $15 \%$ had a temperature of $57000 \mathrm{~K}$, which we suggested might arise from a viscously-heated differentially rotating atmosphere produced by the outburst. Other possible mechanisms for the cooling include compressional heating of outer portions of the WD by the weight of the matter accreted during outburst (Sion 1995) and the effects of a self-sustaining coronal flow onto the surface of the WD during (the early stages) of quiescence (Meyer \& Meyer-Hofmeister 1994).

Our observation of U Gem on Astro-2 occurred at a time when U Gem had been at optical minimum for 185 d (Long, Blair \& Raymond 1995). As shown in Fig. 2, the Astro-2 spectrum of U Gem resembles closely the spectrum obtained on Astro-1. However the flux observed on Astro-2 is less $(70 \%$ at $1450 \AA)$, the color temperature is lower, and the Lyman lines all have larger equivalent widths than on Astro-1. On Astro-2, the best fit single-temperature model was obtained with a temperature of $29700 \mathrm{~K}$ and the flux observed corresponds to a WD radius of $5.410^{8}(\mathrm{D} / 90 \mathrm{pc}) \mathrm{cm}$, consistent with that expected from a $1.05 \mathrm{M}_{\odot}$ WD. These results are similar to those obtained with the Faint Object Spectrograph on Hubble Space Telescope $\sim 13$ and $70 \mathrm{~d}$ after outburst in which the flux at $1450 \AA$ dropped $28 \%$ and in which single-temperature solar abundance model fits indicate a temperature drop from $39400 \mathrm{~K}$ to $32100 \mathrm{~K}$ (Long et al. 1994a). 
The other quiescent dwarf nova in our sample that appears to be dominated by the WD, VW Hyi, was observed twice, 12 and $15 \mathrm{~d}$ after a normal outburst. As was true of $\mathrm{U}$ Gem, the spectrum contains broad absorption lines due to the Lyman series of hydrogen and numerous other absorption features which can be identified with transitions which are expected in a moderately-hot WD atmosphere with near solar abundances. Preliminary fits to the data from both spectra (restricted to the spectral range $1150 \ldots 1840 \AA$ ) using WD model atmospheres suggest a temperature of about $18000 \mathrm{~K}$, similar to the value of $18000 \pm 2000 \mathrm{~K}$ obtained by Mateo \& Szkody (1984) from IUE observations and somewhat lower than the temperature of $22000 \pm 1000 \mathrm{~K}$ inferred by Sion et al. (1995) from FOS observations. However, an $18000 \mathrm{~K}$ model underpredicts the observed emission significantly shortward of $1050 \AA$, suggesting a departure from a simple WD atmosphere, which requires additional analysis.

In contrast to U Gem and VW Hyi, the spectra of SS Cyg, YZ Cnc, and WX Hyi are characterized by blue continua on which are superposed broad (typically $10 \AA \mathrm{FWHM}$ ) emission features, which can be identified with resonance lines such as S VI $\lambda \lambda 933,945, \mathrm{O}$ VI $\lambda \lambda 1032,1038, \mathrm{~N}$ V $\lambda \lambda 1239,1243$, Si IV $\lambda \lambda 1394,1403$ and C IV $\lambda \lambda 1548,1551$. The signatures of the WD, namely broad Lyman and narrow metal absorption lines, appear to be absent, suggesting that some other component of the dwarf nova contributes significantly to these spectra. Longward of Ly $\alpha$ all of the HUT spectra of dwarf novae resemble spectra obtained with IUE [see e.g. la Dous (1990)]. Because of the fact that HUT covers the region shortward of Ly $\alpha$, HUT should have been more sensitive to the WD than IUE. If we insist that the WDs dominate the continuum spectra, then they must be quite hot (> $50000 \mathrm{~K}$ for SS Cyg) so that the Lyman and metal lines will be weak enough to hide underneath the emission lines (and airglow features) in the HUT spectra.

The obvious alternative to the WD is a hot region of the disk which dominates the FUV emission of SS Cyg, YZ Cnc and WX Hyi. Unfortunately, there are few models for a hot component in a quiescent disk, the basic problem being of course that a hot portion of the disk should trigger the disk instability. One interesting possibility, recently investigated by Ko et al. (1996) as an extension of their studies of disks in X-ray binaries, is that $\mathrm{X}$-ray and EUV radiation from the vicinity of the WD photoionizes and heats a surface layer on the disk to produce a hot corona. [As discussed by Meyer \& Meyer-Hofmeister (1990), X-ray illumination of a disk does not induce, but tends to stabilize against disk instabilities.] Specifically Ko et al. have carried out self-consistent, non-LTE calculations of the vertical structure of the disk and used their models to predict the emergent line spectrum. Ko et al. compared their model predictions to ob- 


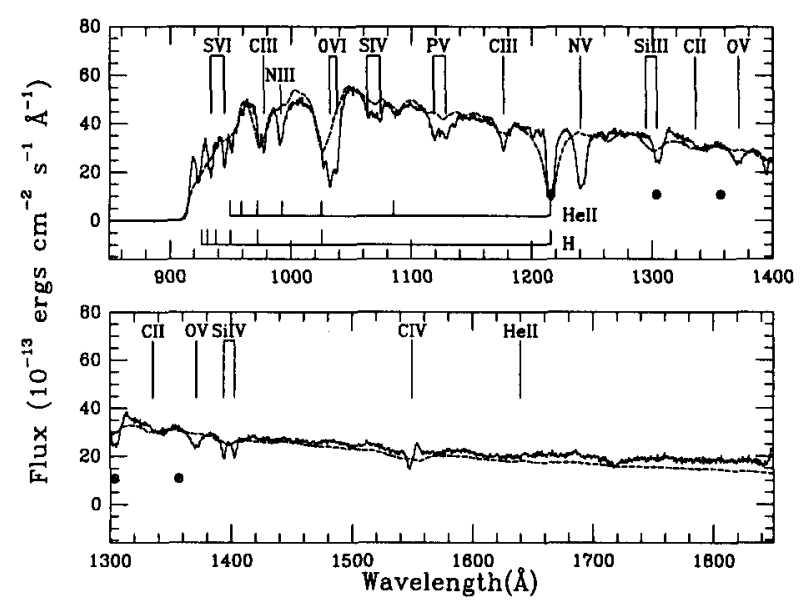

Figure 3. A comparison between the spectrum observed from Z Cam in $1995 \mathrm{March}$, near the peak of an optical outburst and a steady state accretion disk model with $\dot{M}$ of $410^{17} \mathrm{~g} \mathrm{~s}^{-1}$ at a distance of $200 \mathrm{pc}$. The model reproduces the overall spectral shape and flux fairly well, although it is not expected to account for the absorption lines in the wind which produce broad absorption lines of high excitation ions such as O vi $\lambda \lambda 1032,1038$, $\mathrm{N} \vee \lambda \lambda 1239,1243$, and C IV $\lambda \lambda 1548,1551$.

served IUE line fluxes for several dwarf novae, including SS Cyg and WX $\mathrm{Hyi}$, and found that they were able to approximate the line ratios of $\mathrm{N} \mathrm{V}$ $\lambda \lambda 1239,1243, \mathrm{C}$ II $\lambda 1335, \mathrm{Si}$ IV $\lambda \lambda 1394,1403, \mathrm{C}$ IV $\lambda \lambda 1548,1551$, and He II $\lambda 1640$ as long as the X-ray/EUV luminosity of the illuminating source was about $10^{32} \mathrm{erg} \mathrm{s}^{-1}$. The same models appear to be able to account for $\mathrm{C}$ III $\lambda 977$ and $\mathrm{O}$ VI $\lambda \lambda 1032,1038$ line fluxes in the HUT wavelength range as well (Ko 1995 private comm.). It is interesting that the predicted equivalent widths are also close to the equivalent widths we observe, indicating that the photoionized plasma may also contribute to the continuum we observe in these systems.

\section{Observations of optically-thick disks}

In the 'high' state, the FUV spectra of dwarf novae and other similar systems are dominated by the accretion disk as modified by a wind emerging from the inner disk. On Astro-1, HUT was used to observe one dwarf nova in outburst, Z Cam (Long et al. 1991), and two nova-like variables, IX Vel (Long et al. 1994b) and UX UMa. On Astro-2, Z Cam was observed in outburst again (in order to obtain simultaneous coverage with ASCA) and EM Cyg was observed three times (in attempt to follow a dwarf nova through an outburst).

The spectrum of Z Cam on Astro-2 is shown in Fig. 3. It is similar to 


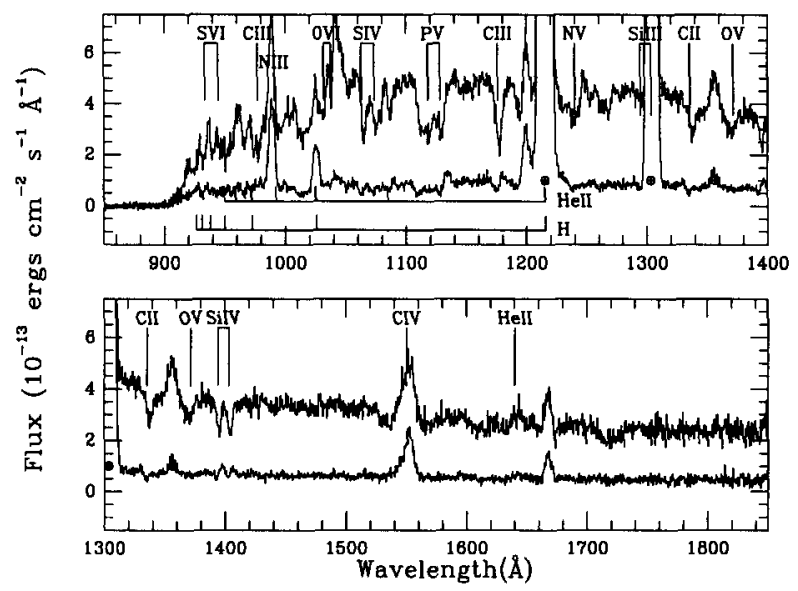

Figure 4. Spectra of EM Cyg obtained during the peak and declining phases of a relatively normal outburst in 1995 March.

the spectrum of Z Cam on Astro-1, and to the spectrum obtained of IX Vel. Prominent absorption features can be identified with the $1 s-2 p$ transitions of Li-like O VI, N V and C IV and of Na-like S VI, P V and Si IV. On Astro-2, the only line in the Z Cam spectrum showing evidence of $\mathrm{P}$ Cygni emission is $\mathrm{C}$ IV $\lambda \lambda 1548,1551$. Presumably this reflects the fact that the $\mathrm{C}$ IV zone in the wind is more extended than that of the other prominent ions in the wind. The spectrum peaks near $1050 \AA$, although it extends to the Lyman limit.

We have attempted fits to the continua of the high state systems observed with HUT using steady-state accretion disk models constructed by summing stellar-model atmospheres and blackbodies. An example of such a fit for Z Cam on Astro-2 is shown in Fig. 3. In these preliminary fits to the data, we assumed a distance of $200 \mathrm{pc}$, an inclination angle of $57^{\circ}$, a WD mass of $0.7 \mathrm{M}_{\odot}, E(B-V)=0.02$ and calculated models for a range of $\dot{M}$. The best-fitting model is shown as the dashed-line in Fig. 3 and provides a reasonable approximation to the continuum shape, including the turnover in the continuum below $1050 \AA$. Similar models constructed with blackbodies would not produce this turnover, which presumably is due to line-blanketing near the Lyman limit.

The spectra from the first and third observations of EM Cyg are shown in Fig. 4. The first spectrum was obtained near the peak of an optical outburst of the system, when the magnitude reported by the AAVSO was about 12.3; the second spectrum was obtained during the decline at magnitude 13.7. Longward of $1000 \AA$ the continuum spectra are very similar aside from an overall decline in flux of about a factor of 5 ; shortward of $1000 \AA$ 
the decline is greater indicating that the peak temperature in the disk has dropped. In the first spectrum, $\mathrm{O}$ VI $\lambda \lambda 1032,1038, \mathrm{~N} \vee \lambda \lambda 1239,1243$ and C IV $\lambda \lambda 1548,1551$ appear to have the absorption and red-side emission features expected from a wind. In the second spectrum, $O$ VI $\lambda \lambda 1032,1038$ and $N \vee \lambda \lambda 1239,1243$ have almost disappeared and $\mathrm{C}$ IV $\lambda \lambda 1548,1551$ is purely in emission. Qualitatively, it is as if the effective size of the disk in the FUV has dropped by about a factor of two and the ionizing flux has declined at the energies necessary to produce $\mathrm{O}$ vi $\lambda \lambda 1032,1038$ and $\mathrm{N} v \lambda \lambda 1239,1243$. Our initial attempts to model the disk spectra with steady state disks have been reasonably successful. For a distance of $300 \mathrm{pc}$, a model spectrum with an $\dot{M}$ of $310^{16} \mathrm{~g} \mathrm{~s}^{-1}$ matches the overall shape of the first spectrum best, although the predicted flux is too small by a factor of 5 . If one demands that the flux be correct, the implied $\dot{M}$ is larger, $210^{17} \mathrm{~g} \mathrm{~s}^{-1}$, but the model spectrum is bluer than is observed.

These problems are similar to those we encountered in attempting to fit the spectrum of the nova-like variable IX Vel from Astro-1, in which the 'best' fitting model (in the sense of approximating the spectral shape) produced had a flux a factor of 5 times lower than required, if the true distance to IX Vel is $95 \mathrm{pc}$. [Similar problems have have discussed by Wade (1988) based on the IUE color indices $\log \left(f_{\lambda 1460} / f_{\lambda 1800}\right)$ and $\log \left(f_{\lambda 1800} / f_{\lambda 2800}\right)$ of nova-like variables.] For IX Vel, as is likely to be true of EM Cyg, improved fits to the data, accounting for both the flux and the shape of the spectrum, could be obtained by modifying the accretion disk structure within 3 WD radii. However, it will be quite difficult to determine whether modifications of this sort represent real physical departures from a steady state disk until more progress is made in constructing models of disks which incorporate not only the vertical structure of the disk, but also radial momentum and energy flow, and perhaps most importantly the disk wind.

\section{Conclusions}

The spectra obtained with HUT on Astro-1 and Astro-2 are the first set of spectra of dwarf novae with sufficient spectral resolution to resolve individual absorption and emission lines in the wavelength range between Ly $\alpha$ and the Lyman limit. The analysis which has been done thus far demonstrates the importance of spectral coverage in this range for CVs, since models that fit the data in the IUE and HST wavelength ranges vary dramatically in the region shortward of Ly $\alpha$. Much more work needs to be done in terms of computing realistic models for comparison with the data, especially to understand the non-WD contributions to the spectra of quiescent dwarf novae and to elucidate the physics of high state accretion disks.

Acknowledgments. The Astro observations of CVs would not have been 
possible without the dedicated support of Janet Mattei and the observers of the AAVSO, my colleagues on the HUT team, especially William Blair and John Raymond who are working with me to analyze the HUT CV data, and the NASA shuttle mission operations and support crew. This work is supported by NASA contract NAS 5-27000 to the Johns Hopkins University.

\section{References}

Baptista, R., Horne, K., Hilditch, R.W., et al., 1995, Ap. J., 448, 395

Cannizzo, J.K., Ghosh, P., Wheeler, J.C., 1982, Ap. J., 260, L83

Córdova, F.A., 1995, in "X-ray Binaries", eds. W. H. G. Lewin, J. van Paridijs, E.P.J. van den Heuvel, Cambridge: Cambridge Univ. Press, p331

Córdova, F.A., Howarth, I., 1987, in "Exploring the Universe with the IUE Satellite", eds. Y. Kondo, et al. D. Reidel, Dordrecht, p395

Davidsen, A.F., Long, K.S., Durrance, S.T., et al., 1992, Ap. J., 392, 264

Drew, J E., 1990, in IAU Colloquium 122, "Physics of Classical Novae", eds. A Cassatella, R. Viotti, Springer-Verlag, Berlin, p228

Horne, K., Marsh, T.R., Cheng, F.H., et al., 1994, Ap. J., 426, 294

Kiplinger, A.L., Sion, E.M., Szkody, P., 1991, Ap. J., 366, 569

Ko, Y., Lee, Y.P., Schlegel, E.M., Kallman, T.R., 1996, Ap. J., 457, 363

Kruk, J.W., Durrance, S.T., Kriss, G.A., et al., 1995, Ap. J., 454, L1

la Dous, C., 1990, Sp. Sci. Rev., 52, 203

Long, K.S., Blair, W.P., Davidsen, A.F., et al., 1991, Ap. J., 381, L25

Long, K.S., Blair, W.P., Bowers, C.W., et al., 1993, Ap. J., 405, 327

Long, K.S., Blair, W.P., Raymond, J.C., 1995, Ap. J., 454, L39

Long, K.S., Sion, E.M., Huang, M., Szkody, P., 1994a, Ap. J., 424, L49

Long, K.S., Wade, R.A., Blair, W.P., et al., 1994b, Ap. J., 426, 704

Mason, K.O., Drew, J.E., Córdova, F.A., et al., 1995, MNRAS, 274, 271

Mateo, M., Szkody, P., 1984 AJ, 89, 863

Meyer, F., Meyer-Hofmeister, E., 1982, A\&A, 106, 34

Meyer, F., Meyer-Hofmeister, E., 1990, A\&A, 239, 214

Meyer, F., Meyer-Hofmeister, E., 1994, A\&A, 288, 175

Michaud, G., 1987, in IAU Colloqoquium 95, "Second Conference on Faint Blue Stars", eds A.G.D. Phillip, D.S. Hayes, J.W. Liebert (Schenectady: Davis), p249

Panek, R.J., Holm, A.V., 1984, Ap. J., 277, 700

Polidan, R.S., Mauche, C.W., Wade R.W., 1990, Ap. J., 356, 211

Raymond, J.C., Mauche, C.W., Bowyer, S., Hurwitz, M., 1995, Ap. J., 440, 331

Robinson, E.L., Wood, J.H., Bless, R.C., et al., 1995, Ap. J., 443, 295

Sion, E.M., 1995, Ap. J., 438, 876

Sion, E.M., Szkody, P., Cheng, F., Huang, M., 1995, Ap. J., 444, L97

Wade, R.A., 1988, Ap. J., 335, 394 